\title{
Heterogeneity of Frailty Trajectories and Associated Factors in the Lothian Birth Cohort 1936
}

\author{
Miles Welstead ${ }^{a, b, c}$ Michelle Luciano ${ }^{a}$ Tom C. Russ ${ }^{a, b}, c$ \\ Graciela Muniz-Terrerab \\ aLothian Birth Cohorts, School of Philosophy, Psychology \& Language Sciences, University of Edinburgh, Edinburgh,

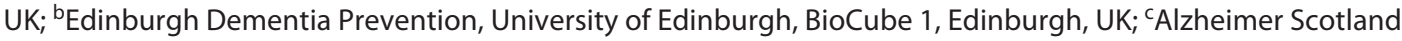 \\ Dementia Research Centre, University of Edinburgh, Edinburgh, UK
}

\section{Keywords}

Latent class mixed models · Subpopulations · Differential ageing $\cdot$ Longitudinal study $\cdot$ Older adults

\begin{abstract}
Introduction: Recent research suggests that the experience of frailty progression may be heterogeneous, with latent subpopulations of older adults following distinct trajectories of frailty. We aimed to investigate this notion and determine whether certain factors are associated with the membership of these subpopulations. Methods: Data from 5 data waves collected over 12 years in participants of the Lothian Birth Cohort 1936, aged 70 at baseline, were used to derive the frailty index (FI) (NW1 = 1,091, NW5 = 431). These were used in latent class mixed modelling to estimate subpopulations of frailty trajectories. Results: A quadratic latent class mixed model found 3 distinct groupings, which followed a low $(61 \%, n=632)$, medium $(36 \%, n=368)$, or high $(3 \%, n=28)$ FI trajectory. Each grouping had different intercepts and slopes, with the high grouping following the steepest trajectory indicating a rapid increase in frailty. Findings showed that in general, those in the low grouping were younger, had higher education, higher age 11 cognitive ability, and were from a higher social class than those in the medium and high
\end{abstract}

karger@karger.com www.karger.com/ger

Karger $\stackrel{\text { ' }}{5}$
(C) 2021 The Author(s)

Published by S. Karger AG, Basel

This is an Open Access article licensed under the Creative Common Attribution-NonCommercial-4.0 International License (CC BY-NC) (http://www.karger.com/Services/OpenAccessLicense), applicable to the online version of the article only. Usage and distribution for commercial purposes requires written permission. groupings. Discussion/Conclusion: Our findings demonstrate heterogeneity in frailty trajectories over 12 years in individuals aged 70 years at baseline. Membership of higher frailty trajectory groupings was associated with lower social class, less education, and lower childhood cognitive ability, indicating the potential for future interventions to target individuals who are at the greatest risk of belonging to the high frailty trajectory. Future research is required to continue this line of inquiry by exploring other risk and protective factors, and importantly, to assess whether it is possible to realign an individual's membership to a less detrimental grouping of frailty trajectory.

(c) 2021 The Author(s). Published by S. Karger AG, Basel

\section{Introduction}

Frailty is characterized by an increased vulnerability to external stressors and a greater risk of disease, disability, and death [1]. It is well established that frailty can affect groups of older people of the same age in different ways [2], and has been shown to be a more reliable indicator of adverse health outcomes than chronological age $[3,4]$. Identifying the ways in which frailty affects different groups in different ways at a cross-sectional level is im- 
portant; however, equally important is exploring how longitudinal frailty trajectories differ across different groups.

A recent systematic review of longitudinal frailty studies [5] found that there is limited evidence of heterogeneity in the field, with most studies exploring mean change over time. For example, previous studies have assessed frailty progression over time accounting for predictive factors such as gender [6], physical activity [7], and inflammation levels [8]. While using a population's mean change in frailty is widely used and effective, it does not always capture the full heterogeneity of a dataset as it estimates the population mean frailty curve under the assumption that all individuals follow a similar pattern of change [9]. Latent class mixed modelling [10], which makes the assumption that there are underlying exclusive latent classes [11], may allow us to better explore the heterogeneity of frailty in a population and isolate frailty trajectories of any underlying subpopulations with distinct patterns of change over time. Identifying subpopulations of frailty change and exploring the factors which may be associated with these trajectories has important implications. Previous studies that have identified distinct frailty trajectories in older adult populations have highlighted the potential role this research may have in targeting high-risk individuals [12]. Understanding these distinct trajectories also improves the ability to design individualized interventions focussed on preventing or delaying the development of numerous adverse outcomes that are associated with frailty [13].

Only a handful of studies using continuous frailty measures have utilized mixture modelling approaches to identify latent trajectories of frailty [5]. Methods of measuring frailty in these studies differed, with most favouring the frailty index (FI), which characterizes frailty as the accumulation of deficits across multiple body systems encompassing physical, social, and cognitive domains [6, 14-16]. For example, FI trajectories were assessed in an English cohort of participants using electronic primary care records [17]. Over the course of 1 year with 12 time points, a latent class mixed model with 3 subpopulations was found to be the best fit (labelled as rapidly rising, moderately increasing, and stable). Comparatively, other studies used a different method to measure frailty, favouring instead the Fried phenotype which measures frailty according to 5 criteria thought to reflect the affected systems of frailty: weight loss; exhaustion; weakness; slowness whilst walking; and low levels of physical activity [18]. For example, 1 longitudinal study [19] used a modified version of the Fried phenotype and found 3 distinct subpopulations in a Mexican-American population over the course of 12 years (labelled as a consistently low group, a progressive moderate group, and a progressive high group).

Although these findings suggest that frailty progression can affect different subpopulations in different ways, further studies are needed [5]. It is important to understand the unique factors associated with these subpopulations, that is, which individuals are more likely to be members of a rapidly rising trajectory opposed to a slow and steady trajectory. Accordingly, here we explored the subpopulations of trajectories in the FI over approximately 12 years in the Lothian Birth Cohort 1936 (LBC1936). FI was used as the measure of frailty as it is measured on a continuous scale, allowing more subtle changes in frailty over time to be detected than a categorical approach [8]. Additionally, it is widely used in the field, allowing comparisons to other studies. We used latent class mixed models to establish a best fitting number of subpopulations, and then explored factors predictive of these subpopulations. In line with previous research $[17,19,20]$, we expected to find at least 3 subpopulations of FI trajectories in the LBC1936. We also expected that those individuals allocated to the most detrimental trajectories will have higher rates of the frailty trajectory risk factors that we are already familiar with such as lower education $[19,21]$, social class [22], and childhood intelligence [23].

\section{Materials and Methods}

\section{Study Sample}

The LBC1936 is a study based upon the follow-up of participants of the Scottish Mental Survey in 1947 which recruited 70,805 Scottish school children all born in 1936 [24]. The first wave of LBC1936 ran from 2004 to 2007 and consisted of 1,091 participants (mean age, standard deviation $[\mathrm{SD}]=69.5[0.8]$ ). Follow-up testing occurred approximately every 3 years thereafter: wave 2 from 2007 to $2010(n=866$, mean age [SD] $=72.5$ [0.7]), wave 3 from 2011 to $2013(n=697$, mean age [SD] $=76.3$ [0.7]), wave 4 from 2014 to $2017(n=550$, mean age $[\mathrm{SD}]=79.3$ [0.6]), and wave 5 from 2017 to $2019(n=431$, mean age [SD] $=82.0$ [0.5]) [25]. Summary data are reported in Table 1 . The study followed the Declaration of Helsinki guidelines and obtained ethical permissions from the Multicentre Research Ethics Committee for Scotland (MREC/01/0/56), Lothian Research Ethics Committee (LREC/2003/2/29), and Scotland A Research Ethics Committee (07/MRE00/58). Written consent was obtained from all participants.

Frailty Measure

A FI was derived at each of the 5 waves of the LBC1936 according to standard guidelines [16]. In total, 30 deficits covering psy- 
Table 1. Summary characteristics of participants at each LBC1936 wave

\begin{tabular}{llllll}
\hline Variables & Wave 1 & Wave 2 & Wave 3 & Wave 4 & Wave 5 \\
& $2004-2007$ & $2007-2010$ & $2011-2013$ & $2014-2017$ & $2017-2019$ \\
\hline Participants, $n$ & 1,091 & 866 & 697 & 550 & 431 \\
Lost to follow-up since previous wave, $n$ & 0 & 225 & 169 & 147 & 119 \\
Age, years, mean (SD) & $69.5(0.8)$ & $72.5(0.7)$ & $76.3(0.7)$ & $79.3(0.6)$ & $82.0(0.5)$ \\
Female, $n$ (\%) & $543(49.8)$ & $418(48.3)$ & $337(48.4)$ & $275(50)$ & $222(52)$ \\
Type of residence, $n$ (\%) & & & & & \\
$\quad$ Own home & $987(90.5)$ & $791(91.3)$ & $637(91.4)$ & $506(92.0)$ & $400(92.8)$ \\
$\quad$ Rented accommodation & $89(8.2)$ & $67(7.7)$ & $49(7.0)$ & $32(5.8)$ & $24(5.6)$ \\
$\quad$ Residential home & $6(0.5)$ & $6(0.7)$ & $2(0.3)$ & $1(0.2)$ & $1(0.2)$ \\
$\quad$ Nursing home & $4(0.4)$ & $0(0.0)$ & $0(0.0)$ & $0(0.0)$ & $0(0.0)$ \\
$\quad$ Other & $5(0.4)$ & $2(0.2)$ & $9(1.2)$ & 11 & $6(1.4)$ \\
FI, mean (SD) & $0.16(0.1)$ & $0.18(0.1)$ & $0.20(0.1)$ & $0.21(2.0 \%)$ & $0.22(0.1)$ \\
Social class, $n$ (\%) & & & & & \\
$\quad$ Professional & $194(17.8)$ & $170(19.6)$ & $144(20.7)$ & $127(23.1)$ & $102(23.7)$ \\
$\quad$ Managerial & $412(37.8)$ & $331(38.2)$ & $271(38.9)$ & $217(39.5)$ & $171(39.7)$ \\
$\quad$ Skilled nonmanual & $249(22.8)$ & $188(21.7)$ & $143(20.5)$ & $109(19.8)$ & $93(21.6)$ \\
$\quad$ Skilled manual & $191(17.5)$ & $144(16.6)$ & $111(15.9)$ & $78(14.2)$ & $52(12.1)$ \\
$\quad$ Semiskilled/unskilled & $45(4.1)$ & $33(3.8)$ & $28(4.0)$ & $19(3.5)$ & $13(3.0)$ \\
Age 11 cognitive ability, mean (SD) & $0.0(11.6)$ & $0.5(11.8)$ & $1.2(11.8)$ & $1.4(11.8)$ & $1.8(11.6)$ \\
Years of education, mean (SD) & $10.7(1.1)$ & $10.8(1.1)$ & $10.8(1.1)$ & $10.9(1.2)$ & $10.9(1.2)$
\end{tabular}

$\mathrm{FI}$, frailty index; SD, standard deviation.

chological, cognitive, and physical dimensions were used to construct the measure. A full list of the included deficits and their cutoff values has been described in more detail previously [8]. To calculate an FI score for each participant, their present deficits were summed and divided by the number of total deficits $(n=30)$. For instance, a participant with 12 deficits would have an FI score of $12 / 30=0.4$. Scores ranged from 0 to 1 with a higher score indicating a higher level of frailty.

\section{Covariates}

Covariates were included to assess differences in subpopulation characteristics. We included: age at baseline, sex, years of education, age 11 cognitive function, and social class (professional/ managerial/skilled, nonmanual/skilled manual or semiskilled/unskilled). Age 11 cognitive function was derived as part of the LBC1936 using the Moray House Test at age 11 [24] and standardized into an IQ-type score for age in days on the test date. Social class was derived from the principal occupation of each participant and coded using the 1980 census [26]. More detail has been reported in previous LBC1936 articles $[8,25]$.

\section{Missing Data}

Across the 5 waves of the LBC1936 there were instances where some of the items needed to derive the FI were missing. To address this, we used multiple imputations using the MICE package in $\mathrm{R}$ version 4.0.3 to impute these values $[27,28]$. Five rounds of imputations estimated the missing data and a total of 49 missing values $(4.5 \%)$ were replaced with substituted values. Full details of this are published elsewhere [8].

Heterogeneity of Frailty Trajectories

\section{Statistical Analyses}

In order to identify latent subpopulations of FI trajectories in the LBC1936, latent class mixed models were used. Of the 1,091 participants, 63 had missing covariate data. Accordingly, 1,028 participants were included in the latent class mixed models. To establish which model was best fitting for the data, it was necessary to compare models describing different functional shapes for the frailty trajectories. First, models describing linear rate of change and with an increasing number of classes were estimated. Next, we estimated models describing quadratic change with an increasing number of classes. The best fitting model was identified comparing values for Akaike information criterion (AIC) and Bayesian information criterion (BIC) [29], indices that help identify the most parsimonious model. Models with the lowest AIC and BIC are preferred. Posterior probabilities were calculated for each participant, and participants were assigned to a certain trajectory group according to the class with the highest probability [30]. As a measure of fit, average posterior probabilities were calculated and reported.

Comparisons of the AIC and BIC of different models revealed that a quadratic model with either 3 or 4 classes of FI trajectories showed the best fit. A model with 4 classes of latent FI trajectories showed a marginally better fit according to the BIC value $(-9,995.12)$ and AIC value $(-10,105.01)$ than a 3 -class model (BIC: $-9,979.04$, AIC: $-10,068.94)$. However, the 4-group model had relatively low posterior probabilities for each of the group memberships ( 0.71 in 2 cases), whilst the 3 -group model showed posterior probabilities above 0.83 (group 1: 0.90 , group 2: 0.84 , group 3: 0.95). Typically probabilities above 0.80 indicate a strong fitting model [31], and accordingly, the posterior probabilities indicate that discrimination of individuals into groups in the 4-group mod- 


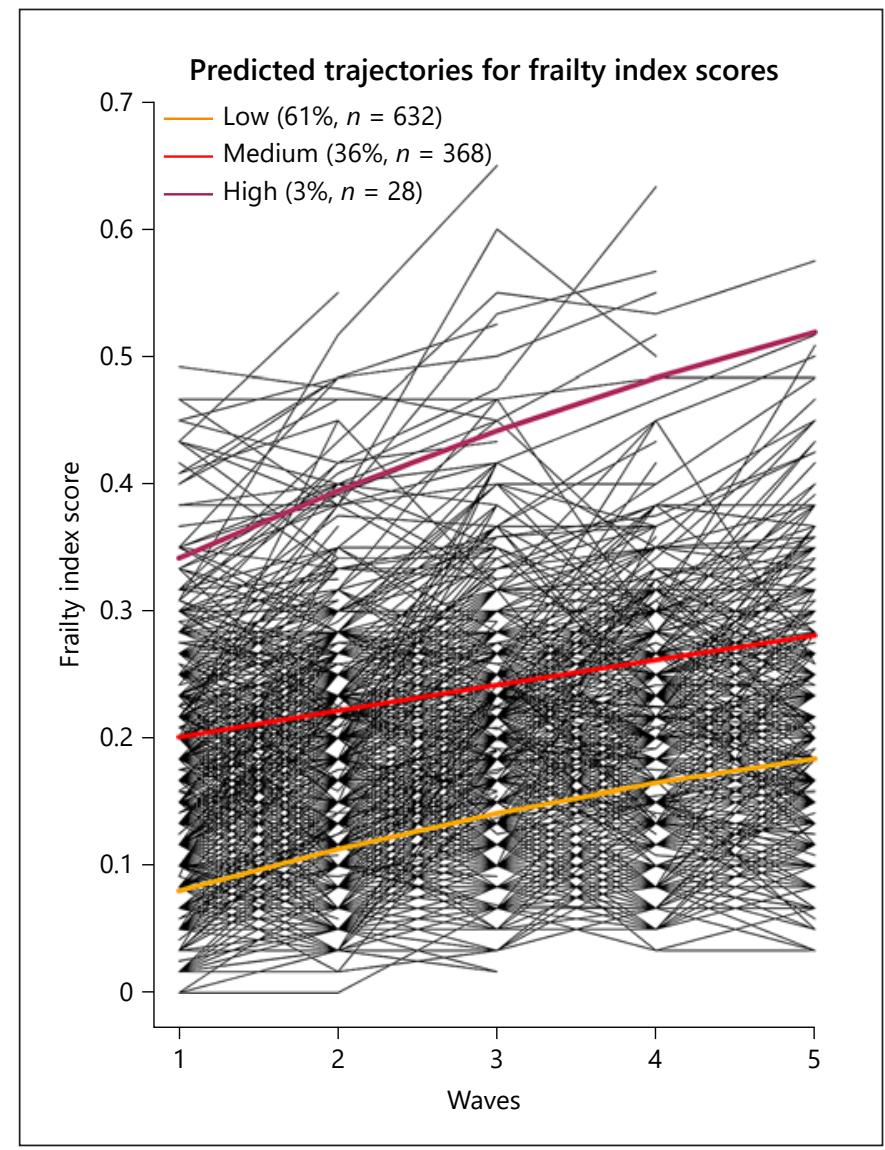

Fig. 1. FI scores for each individual of the LBC1936 over the 5 waves. Three trend lines show differing trajectories for different subpopulations. FI, frailty index.

el was fuzzier than in the 3-group model. Additionally, upon further inspection, all individuals assigned to the smallest grouping in the 4-group model were also assigned to the smallest grouping in the 3-group model. Subsequently, taking into account each of these indicators, preference was given to the 3 group quadratic model, as it was the most parsimonious. AIC and BIC values for each model are reported in online suppl. Table 1; for all online suppl. material, see www.karger.com/doi/10.1159/000519240. Comparisons of the covariates across different trajectory groupings were assessed by ANOVAs, $t$ tests, and Pearson's $\chi^{2}$ tests. All analyses and figure creation were undertaken in $\mathrm{R}$ Version 3.6.1 [28] with the latent class mixed models using the Hlme package, and Figure 1 using the Spaghettiplot function.

\section{Results}

Of the 1,028 participants with complete data, 520 (51\%) were male and 508 (49\%) were female. The mean age of these participants at baseline was 69.58 (SD 0.84).
Using a quadratic latent class mixed model, 3 groupings of latent FI trajectories were identified. As illustrated in Figure 1, findings show 3 distinct FI trajectories with differing intercepts and slopes. For ease of understanding, these 3 trajectory groups will henceforth be referred to as groups Low, Medium, and High.

In the quadratic model the intercept represented the baseline FI, the linear slope corresponded to the rate of change over time, and the quadratic term indicated the change in the rate of change, that is, the acceleration or deceleration over time. The low grouping was most common with $61 \%(n=632)$ of participants. Participants in this grouping showed a baseline FI of $0.08(\mathrm{SE}=0.01)$ with a linear slope of $0.04(\mathrm{SE}=0.00)$. However, the quadratic term indicated that this increase significantly slowed over the course of the 5 waves $(q=-0.002)$. Accordingly, by the final wave the low grouping had increased by 0.10 to a FI score of 0.18 . The medium grouping contained $36 \%(n=368)$ of the sample, showing a baseline FI of $0.20(\mathrm{SE}=0.01)$ with a linear slope of 0.02 $(\mathrm{SE}=0.01)$. By the final wave, the medium grouping had increased by 0.08 to a FI score of 0.28 . The high grouping held only a small minority of the sample with the remaining 3\% $(n=28)$. This grouping showed the highest baseline FI of 0.34 ( $\mathrm{SE}=0.03)$ and the steepest trajectory with a linear slope of $0.06(\mathrm{SE}=0.02)$ which equated to an overall increase of 0.18 by the final wave to a FI score of 0.52 .

\section{Probability of Classification}

Results estimating the probability of group membership as a function of age, sex, education, age 11 cognitive functions, and social class, indicated that there were significant differences between the 3 groups. As reported in Table 2, associations between the covariates and FI trajectory group were significant for age at baseline, social class, age 11 cognitive ability, and years of education. No sex differences were found. Post hoc $t$ tests and Pearson's $\chi^{2}$ tests were used to assess the difference between specific FI trajectory groups and the covariates (low vs. medium/ low vs. high/medium vs. high). Significant differences indicated that members of the low grouping were significantly more likely to come from a higher occupational social class than those in the medium $\left(\chi^{2}[5]=67.67, p<\right.$ $0.001)$ or high grouping $\left(\chi^{2}[5]=51.72, p<0.001\right)$. Those in the low grouping were also significantly younger at baseline than those in the medium grouping $(t[998]=$ $-10.31, p<0.001)$, but not the high grouping. Baseline age did not significantly differ between the medium and high groups. Years of education was significantly higher in the 
Table 2. A comparison of covariate characteristics between latent classes

\begin{tabular}{|c|c|c|c|c|}
\hline Age at baseline, mean (SD) & $69.38(0.83)$ & $69.92(0.75)$ & $69.62(0.74)$ & $<0.001^{1 *}$ \\
\hline \multicolumn{5}{|l|}{ Sex, $n(\%)$} \\
\hline Male & $324(51.3)$ & $182(49.5)$ & $14(50.0)$ & \multirow[t]{2}{*}{$0.857^{2}$} \\
\hline Female & $308(48.7)$ & $186(50.5)$ & $14(50.0)$ & \\
\hline Managerial & $261(41.3)$ & $120(32.6)$ & $7(25.0)$ & \multirow{4}{*}{$<0.001^{2 *}$} \\
\hline Skilled nonmanual & $140(22.2)$ & $92(25.0)$ & $3(10.7)$ & \\
\hline Skilled manual & $68(10.8)$ & $98(26.6)$ & $13(46.4)$ & \\
\hline Semiskilled/unskilled & $18(2.9 \%)$ & $21(5.7 \%)$ & $4(14.3 \%)$ & \\
\hline Age 11 cognitive ability, mean (SD) & $3.95(9.08)$ & $-5.57(11.80)$ & $-15.96(15.30)$ & $<0.001^{1 *}$ \\
\hline Years of education, mean (SD) & $10.99(1.16)$ & $10.29(0.90)$ & $10.14(0.89)$ & $<0.001^{1 *}$ \\
\hline
\end{tabular}

${ }^{*} p<0.001 .{ }^{1}$ Linear model ANOVA. ${ }^{2}$ Pearson's $x^{2}$ test. SD, standard deviation.

low grouping than both the medium $(t[998]=9.98, p<$ $0.001)$ and high grouping $(t[658]=3.82, p<0.001)$. Age 11 cognitive ability was significantly higher in the low grouping than both the medium $(t[998]=14.28, p<$ $0.001)$ and high groupings $(t[658]=10.95, p<0.001)$, and also significantly higher in the medium grouping than the high grouping $(t[394]=4.39, p<0.001)$. No other statistically significant findings between specific FI trajectory groups and covariates were found.

\section{Discussion/Conclusion}

In this study, we investigated the existence of subgroups of individuals with distinct patterns of frailty change over time using data from the LBC1936. Using a growth mixture model, we found that participants of the LBC1936 tend to fit into one of 3 trajectory groups. Of the population, $61 \%$ fit into the low grouping, $36 \%$ into the medium grouping, and 3\% into the high grouping. Each grouping showed significantly different baseline FI scores and differing slopes of change over time. The high grouping showed both the highest baseline FI score and steepest slope of change, indicating a rapid increase in frailty over the 5 waves. Whilst the low and medium groupings have significantly different baseline FI scores, over the 5 waves, they gradually begin to take converge due to a marginally steeper FI trajectory in the low grouping. Further analysis revealed that membership of these 3 groupings of FI trajectory were affected by social class, years of education, age at baseline, and age 11 cognitive ability.

Heterogeneity of Frailty Trajectories
Comparison with Other Literature and Interpretation

Comparisons with similar literature prove difficult due to differing frailty outcome measures and time scales. For example, 2 longitudinal studies $[19,20]$ found 3 distinct frailty trajectories in a group of older adults. However, both of these studies utilized the Fried phenotype, which not only conceptualizes frailty in a different way to the FI, but it also measures frailty as a categorical variable. Comparatively, 1 longitudinal study [17] utilized the FI and similar statistical analyses as our study, finding 3 frailty trajectories. However, this study followed participants in the year preceding the participant's death, meaning that the trajectories will presumably be higher and more pronounced than a relatively healthy older adult sample like the LBC1936. However, despite these substantial methodological differences between previous studies, it is notable that our findings of 3 distinct trajectories are consistent with all of these mentioned studies. This indicates that frailty does seem to affect 3 subpopulations of older adults in different ways. It is unsurprising to observe that those in the low grouping had a steeper FI trajectory than those in the medium grouping as this supports previous findings and the notion that those with the most to lose tend to show steeper slopes of decline. For example, 1 study which identified 3 distinct frailty trajectories [17] found that the grouping with the lowest baseline frailty rate also showed the steepest trajectory.

Our findings indicated that social class, years of education, age at baseline, and age 11 cognitive ability all significantly differed according to FI trajectory grouping. These findings reinforce previous research which impli- 
cates these factors as predictors of frailty, largely finding that younger, more educated individuals from a higher social class are less likely to become frail [5]. These findings are significant as they indicate that modifiable factors such as education may be able to provide an effective way to help reduce an individual's risk of higher levels of frailty. It seems probable that there may be other underlying factors that we have not tested for which help explain trajectory group membership. Further research is required to assess other factors and explore the causality of these in relation to FI trajectory group membership.

Irrespective of the potential factors associated with FI trajectory groupings, it is clear that a certain subpopulation of older adults is at great risk of developing frailty and rapidly declining, presumably until death occurs. By understanding these trajectories and mapping out the pathways to which these subpopulations follow, it will be better possible to put in place effective early interventions and implement frailty treatment measures. A crucial question for future research is whether individuals are able to change between FI trajectory groupings, for example, with early intervention is it possible for an individual in the high grouping to shift into the medium or low grouping. Additionally, it would be highly informative to further explore the associations between frailty trajectory groupings and clinical data such as mortality, disability, and health-care use.

\section{Limitations and Strengths}

There are several limitations to consider in this study. Firstly, it has been well established that the participants of the LBC1936 have higher levels of physical health and cognitive ability than the general population [25, 32]. Physical health and cognitive ability both contribute to frailty risk [5], and accordingly, our results may be conservative when considering the general population whereby a larger proportion may be at risk of falling into the medium or high groupings. Secondly, after splitting the sample into the 3 groupings, the high grouping contained only 28 participants, and accordingly, larger studies are required to validate our results. Thirdly, as with most longitudinal studies in older adults, due to significant rates of attrition or loss to follow-up due to death, LBC1936 has a healthy survivor effect whereby those who remain in the study throughout follow-up are also the participants with better overall health. Previous LBC1936 has shown that those who dropout of the study had lower socioeconomic status, physical fitness, and cognitive ability [25]. Subsequently, this study may also underestimate how steep the frailty trajectories are in the general population.
Fourthly, due to the FI's biopsychosocial approach of including a wide range of variables from various domains, and due to small sample sizes reducing the analysis power, it was not feasible to control some important factors such as physical activity levels, body mass index, or polypharmacy. Accordingly, it may be necessary for future research to undertake a similar analysis in a larger cohort using a frailty measure, such as the Fried phenotype [18] which focusses purely on physical domains, and would subsequently allow for more covariates to be considered. And finally, whilst the FI has been shown to have good reliability and validity as a measure of frailty, there are many different ways of measuring frailty, and it is important to acknowledge that studies like this one which uses the FI may not be comparable to other studies using different frailty measurement tools [33].

This study also had several strengths. The follow-up period was around 12 years with 5 time points (age $\sim 69$ to $\sim 82$ years old). Amongst community dwelling individuals, it is thought that around $10 \%$ of 65 year olds are frail [34], with this percentage increasing significantly over the following decades. With this in mind, our follow-up period allowed us to track frailty in later life over a critical period of frailty progression. Additionally, by utilizing latent class mixed models, we were able to capture the heterogeneity of frailty progression in later life. By doing so, we provide a more individualized approach to frailty, acknowledging that not all groups of people will be the same. This approach has the potential to inform and optimize future prevention strategies with a more targeted approach.

\section{Conclusions}

This study identified 3 trajectories of frailty in the Lothian Birth Cohort 1936 across approximately 12 years of data collection. Low, medium, and high trajectories were found to differ significantly on a number of factors including social class, education, and age 11 cognitive ability. Addressing education and social class disparities may help to close the gap between the most detrimental frailty trajectories and improve health outcomes. Our findings are a preliminary indication of heterogeneity in the progression of frailty in later life. Future research should continue to develop this line of research by implementing consistent frailty measures in different samples and utilizing latent class mixed model analysis to reveal any trajectories of subpopulations. By exploring the heterogeneity of frailty trajectories in different populations, and factoring in variables such as social class, education, and age 
11 cognitive ability, this study sheds further light on how frailty influences certain groups of people over time. Further work into these avenues of research are imperative in furthering our understanding of frailty and informing therapeutic and preventative interventions.

\section{Acknowledgment}

The authors thank all of the LBC1936 participants who have contributed to the study. We also thank the team members for collecting and collating the data that have been used in this study.

\section{Statement of Ethics}

The study followed the Declaration of Helsinki guidelines and obtained ethical permissions from the Multicentre Research Ethics Committee for Scotland (MREC/01/0/56), Lothian Research Ethics Committee (LREC/2003/2/29), and Scotland A Research Ethics Committee (07/MRE00/58). Written consent was obtained from all participants.

\section{Conflict of Interest Statement}

The authors have no conflicts of interest to declare.

\section{Funding Sources}

Age UK (MR/M01311/1) and MRC (G1001245/96099) are involved in funding the recruitment and data collection for the Lothian Birth Cohort 1936. The sponsor had no role in the design, methods, analysis, and preparation of paper.

\section{Author Contributions}

Miles Welstead contributed to conceptualization, methodology, software, formal analysis, writing - original draft, writing - review and editing, and visualization. Graciela Muniz-Terrera contributed to conceptualization, methodology, writing - review and editing, and supervision. Tom C. Russ contributed to conceptualization, methodology, writing - review \& editing, and supervision. Michelle Luciano contributed to conceptualization, methodology, writing - review and editing, and supervision.

\section{Data Availability Statement}

All data generated or analysed during this study are included in this article (and/or) its online suppl. files. Further enquiries can be directed to the corresponding author.

\section{References}

1 Collard RM, Boter H, Schoevers RA, Oude Voshaar RC. Prevalence of frailty in community-dwelling older persons: a systematic review. J Am Geriatr Soc. 2012;60(8):1487-92.

2 Hoogendijk EO, van Hout HP, Heymans MW, van der Horst HE, Frijters DH, Broese van Groenou MI, et al. Explaining the association between educational level and frailty in older adults: results from a 13-year longitudinal study in the Netherlands. Ann Epidemiol. 2014;24(7):538-44.e2.

3 Schuurmans H, Steverink N, Lindenberg S, Frieswijk N, Slaets JP. Old or frail: what tells us more? J Gerontol A Biol Sci Med Sci. 2004; 59(9):M962-5.

4 Romero-Ortuno R, Kenny RA. The frailty index in Europeans: association with age and mortality. Age Ageing. 2012;41(5):684-9.

5 Welstead M, Jenkins ND, Russ TC, Luciano M, Muniz-Terrera G. A systematic review of frailty trajectories: their shape and influencing factors. Gerontologist. 2020 Jun 2. Epub ahead of print.

6 Marshall A, Nazroo J, Tampubolon G, Vanhoutte B. Cohort differences in the levels and trajectories of frailty among older people in England. J Epidemiol Community Health. 2015;69(4):316-21.
7 Rogers NT, Marshall A, Roberts CH, Demakakos P, Steptoe A, Scholes S. Physical activity and trajectories of frailty among older adults: evidence from the english longitudinal study of ageing. PLoS One. 2017;12(2): e0170878.

8 Welstead M, Muniz-Terrera G, Russ TC, Corley J, Taylor AM, Gale CR, et al. Inflammation as a risk factor for the development of frailty in the Lothian Birth Cohort 1936. Exp Gerontol. 2020;139:111055.

9 McCulloch CE, Lin H, Slate EH, Turnbull BW. Discovering subpopulation structure with latent class mixed models. Stat Med. 2002;21(3):417-29.

10 Vermunt JK, Magidson J. Latent class analysis. In: The sage encyclopedia of social sciences research methods. Thousand Oaks, CA: SAGE; 2004. Vol. 2; p. 549-53.

11 Lanza ST, Rhoades BL. Latent class analysis: an alternative perspective on subgroup analysis in prevention and treatment. Prev Sci. 2013;14(2):157-68.

12 Chamberlain AM, Finney Rutten LJ, Manemann SM, Yawn BP, Jacobson DJ, Fan C, et al. Frailty trajectories in an elderly population-based cohort. J Am Geriatr Soc. 2016 Feb;64(2):285-92.
13 Clegg A, Young J, Iliffe S, Rikkert MO, Rockwood K. Frailty in elderly people. Lancet. 2013;381(9868):752-62.

14 Rockwood K, Mitnitski A. Frailty in relation to the accumulation of deficits. J Gerontol A Biol Sci Med Sci. 2007;62(7):722-7.

15 Mitnitski AB, Mogilner AJ, Rockwood K. Accumulation of deficits as a proxy measure of aging. ScientificWorldJournal. 2001;1:32336.

16 Searle SD, Mitnitski A, Gahbauer EA, Gill TM, Rockwood K. A standard procedure for creating a frailty index. BMC Geriatr. 2008; $8(1): 24$.

17 Stow D, Matthews FE, Hanratty B. Frailty trajectories to identify end of life: a longitudinal population-based study. BMC Med. 2018; 16(1):171.

18 Fried LP, Tangen CM, Walston J, Newman $A B$, Hirsch C, Gottdiener J, et al. Frailty in older adults: evidence for a phenotype. J Gerontol A Biol Sci Med Sci. 2001;56(3): M146-56.

19 Peek MK, Howrey BT, Ternent RS, Ray LA, Ottenbacher KJ. Social support, stressors, and frailty among older Mexican American adults. J Gerontol B Psychol Sci Soc Sci. 2012; 67(6):755-64. 
20 Hsu HC, Chang WC. Trajectories of frailty and related factors of the older people in Taiwan. Exp Aging Res. 2015;41(1):104-14.

21 Chen F, Mair CA, Bao L, Yang YC. Race/ethnic differentials in the health consequences of caring for grandchildren for grandparents. J Gerontol B Psychol Sci Soc Sci. 2015;70(5): 793-803.

22 Alvarado BE, Zunzunegui MV, Béland F, Bamvita JM. Life course social and health conditions linked to frailty in Latin American older men and women. J Gerontol A Biol Sci Med Sci. 2008;63(12):1399-406.

23 Gale CR, Booth T, Starr JM, Deary IJ. Intelligence and socioeconomic position in childhood in relation to frailty and cumulative allostatic load in later life: the Lothian Birth Cohort 1936. J Epidemiol Community Health. 2016;70(6):576-82.
24 Penrose L; Scottish Council for Research in Education. The trend of Scottish intelligence: a comparison of the 1947 and 1932 surveys of the intelligence of eleven-year-old pupils. London: University of London Press Ltd; 1949. p. 151+ xxviii. Price 7s. 6d. Ann Eugen. 1949;15(1):186-7.

25 Taylor AM, Pattie A, Deary IJ. Cohort profile update: the Lothian birth cohorts of 1921 and 1936. Int J Epidemiol. 2018;47(4):1042-r.

26 General R. Office of population censuses and surveys. Classification of occupations. London: HMSO; 1991.

27 van Buuren S, Groothuis-Oudshoorn CG. Mice: multivariate imputation by chained equations in R. J Stat Softw. 2010;45(3):168.

28 R Core Team. R: a language and environment for statistical computing. 2018;1(1358):34. $\operatorname{dim}(\mathrm{ca} 533)$.

29 Schwarz G. Estimating the dimension of a model. Ann Stat. 1978;6(2):461-4.
30 Jung T, Wickrama KAS. An introduction to latent class growth analysis and growth mixture modeling. Soc Pers Psychol Compass. 2008;2(1):302-17.

31 Proust-Lima C, Séne M, Taylor JM, JacqminGadda H. Joint latent class models for longitudinal and time-to-event data: a review. Stat Methods Med Res. 2014;23(1):74-90.

32 Deary IJ, Gow AJ, Taylor MD, Corley J, Brett C, Wilson V, et al. The Lothian Birth Cohort 1936: a study to examine influences on cognitive ageing from age 11 to age 70 and beyond. BMC Geriatr. 2007;7(1):28.

33 Aguayo GA, Donneau AF, Vaillant MT, Schritz A, Franco OH, Stranges S, et al. Agreement between 35 published frailty scores in the general population. Am J Epidemiol. 2017;186(4):420-34.

34 Kojima G, Liljas AEM, Iliffe S. Frailty syndrome: implications and challenges for health care policy. Risk Manag Healthc Policy. 2019; 12:23. 